\title{
El valor de las acciones ambientales y su evidencia en el contexto formativo
}

\author{
Liliana Caycedo ${ }^{1}$
}

Sonia Maria Rosas ${ }^{2}$

\begin{abstract}
Resumen
Las acciones y las actividades ambientales son términos propios del lenguaje de la educación ambiental; sin embargo, es pertinente precisar que las primeras tienen transcendencia sobre el currículo; mientras que las actividades ambientales pueden estar desligadas de este.
\end{abstract}

La formación ambiental no puede limitarse exclusivamente al compendio conceptual sino que debe trascender a lo más íntimo de las personas para transformar con intención el componente axiológico y afectivo sin dejar de lado la rigurosidad de los conceptos naturales y científicos que le atañen a su propia naturaleza. El descuido de cualquiera de las partes: conceptual, actitudinal, afectiva ó axiológica desencadena lo que hemos denominado activismo ambiental.

Palabras clave: acción ambiental, actividad ambiental, formación e información ambiental. 


\title{
The value of environmental actions and evidence about them in an educational contexts
}

\begin{abstract}
Environmental actions and activities are terms belonging to the language of environmental education. However, it is worth pointing out the first ones have an incidence on curriculum, while environmental activities may be unrelated to curriculum.

Environmental education needs not to be constrained to the conceptual précis, but it must transcend to the most intimate in people in order to purposedfully transform the axiological and affective component, without leaving aside the rigorousness of natural and scientific notions inherent to them. Overlooking any dimension of it: the conceptual, attitudinal, affective or axiological results in what we have called environmental activism.
\end{abstract}

Key Words: environmental action, environmental activity, environmental formation and information.

De acuerdo con Gomera (2008), la conciencia ambiental aborda dos dimensiones que se pueden involucrar con la intención de propiciar acciones ambientales. La primera corresponde a la dimensión cognitiva, entendida como la disposición para adoptar criterios proambientales en la conducta, manifestando interés o predisposición a participar en actividades y aportar soluciones. La segunda, por su parte, se refiere a una dimensión activa que refleja la realización de prácticas y comportamientos ambientalmente responsables, tanto individuales como colectivos.

En el estudio de Gomera (2008), desarrollado en el ámbito universitario, se analiza la diferencia entre acciones ambientales relacionadas con la trascendencia sobre el mismo currículo y las actividades ambientales desligadas de este. De las últimas se puede afirmar que no ofrecen formación una vez realizadas, pues su único fin es el de cumplirse dentro de un plan definido en educación ambiental, sin estar necesariamente direccionadas 
hacia la transformación del individuo en la percepción y aceptación de su responsabilidad ambiental.

En relación al currículo, y tomando los argumentos teóricos de Elliot (---), se pueden definir tres tipos cuya influencia resulta válida en el acondicionamiento de la formación ambiental. Primero está el llamado currículo explícito, que define estándares y objetivos; por otro lado está el currículo implícito, en donde se evidencian las interacciones de docentes y estudiantes, y que, por esa misma interacción, genera debate y crítica; y finalmente un currículo nulo, en donde no hay comunicación ni posibilidad de debate, la información es escasa y no es incluyente.

En ese sentido, las acciones ambientales deberían enmarcarse en los dos primero tipos de currículo. Sin embargo, frente a la formación ambiental no sucede de esta manera. Probablemente porque se parte del argumento de formar en lo natural a quienes pertenecen a la misma naturaleza y por tal razón parece generarse un debate en donde el hombre es el centro de la acción ambiental, pero influenciado más por factores políticos y económicos que naturales.

Chawla (1999) ya advertía al respecto de esta condición en la formación ambiental cuando afirma que las acciones a favor del medio ambiente tienen un encuentro definitivo en la infancia, especialmente por el entorno en el cual el niño recibe una influencia marcada de quienes le rodean. En ese sentido, la formación ambiental y las acciones ambiéntales positivas derivadas de aquella no pueden ligarse a la formalidad de las aulas y los currículos planeados, sino que están claramente influenciados por una historia de sucesos afortunados o desafortunados que desde la infancia, se relacionan con la conservación y cuidado del medio ambiente.

Ahora bien, si se piensa en la influencia de la edad para el desarrollo de las acciones ambientales y/o su apropiación a través de una formación planeada, es interesante revisar la propuesta asociativa que hace García (2005) acerca de los principios Vygotskianos de Steiner, enfocados principalmente en el desarrollo emocional de los individuos creativos. La propuesta de García (2005) está apoyada además en las investigaciones de Frawley (1999), de quien cita:

En el pensamiento, cuando se superpone con el discurso y se convierte en algo mediado, no existe nada esencialmente adulto o infantil, ya que todos tenemos el mismo problema de manejo de la individuación respecto al grupo(García 2005:27).

Entonces la acción ambiental, más allá de la respuesta al currículo, se puede identificar cuando el individuo y su colectivo responden al reflejo de las dimensiones antes mencionadas. Si además se entiende que el fin 
de toda acción de educación ambiental es facilitar aun más la resolución de un determinado problema ambiental y/o la comprensión de lo ambiental, entonces la ambientalización del currículo no es suficiente para generar esta competencia en el estudiante. Son en cambio las interacciones ambientales previas y el diagnóstico preciso de la conciencia ambiental de cada individuo lo que definirá a futuro el tipo de acción y actividades que deben planearse para no perder el sentido de la educación ambiental.

En el campo de la educación superior las acciones ambientales se dimensionan no solo desde las plataformas estratégicas, sino que se convierten en propositivas para el cumplimiento en los tres aspectos misionales que desarrollan. Un ejemplo de acción ambiental colectivo es la reducción en el consumo de agua de una institución; si bien la acción es un deseo amplio, las actividades individuales resultado de la educación y el compromiso con una política ambiental logran que dicho consumo alcance los niveles deseados y que el colectivo sin ningún tipo de presión transforme la cultura ambiental. Pero además de esto, la universidad debería generar desde la investigación, desarrollos tecnológicos que permitan la optimización del uso del recurso agua y por otro lado la orientar la docencia hacia la conciencia teórica del uso responsable de este recurso.

En un caso como el anterior se puede hablar de una orientación ambiental, decidida y operante, y no solamente de actividades y lecciones teóricas relacionadas con lo ambiental; cuando se logra esta integración se traza el camino hacia una formación ambiental respaldada por las acciones ambientales y viceversa. La discusión respecto a las acciones podría girar siempre en el mismo registro, que es la capacidad adquirida de modificar los hábitos y la comprensión de la dimensión ambiental propia. Sin embargo, para dar un poco más de ilustración, en este artículo se definirán algunas variables asociadas a la inducción y transformación de los comportamientos, que a su vez generan acciones ambientales con compromiso.

\section{Información y formación ambiental.}

De acuerdo con Jaén (2009) quien cita a Kollmuss y Agyeman (2002), existe en la sociedad actual más información e incluso mayor reconocimiento de la historia, pero un mínimo de disposición para la modificación de estilos de vida. Frente al exceso de información, la formación se debe tomar como herramienta de modelado para el cambio, entendido dentro de una didáctica y acompañado de un reconocimiento de las experiencias propias: las que plantea el colectivo y las que requiere el medio ambiente. Sin embargo, cuando se enmarca la información y la formación en las acciones ambientales, se genera una línea invisible que no permite una diferenciación clara.

Tradicionalmente el principal pretexto de diferenciación entre información y formación es el que está sujeto a las acciones derivadas de la educación ambiental 
formal y la no formal. Castro (2000) le adjudica a la educación no formal atributos de un proceso intencional, estructurado y sistemático. Si se piensa en lo formal, la diferencia radica en que ésta última se desarrolla en un currículo y una institución definida bajo los parámetros propios de filosofía y autonomía.

Así, la educación ambiental tiene la oportunidad de ampliar su cobertura fuera de las instituciones y llegar a todos los miembros de la sociedad por un mecanismo no formal. Dicha oportunidad ha sido desaprovechada por las mismas instituciones educativas y gubernamentales que pueden a través de la proyección social mejorar sus entornos y direccionar las acciones ambientales hacia la formación.

La discusión en relación a esta diferencia es amplia sin embargo, para el caso que nos ocupa respecto a las acciones ambientales resulta relevante decir quela educación formal conserva un imperativo que genera una «ciudadanía» en la que se identifica al individuo con el colectivo por las características de convivencia a mediano y largo plazo, propias de las instituciones que la ofrecen. Entre tanto, la educación informal involucra un corto plazo que no permite generar identidad con una cultura inherente a los valores y principios de actuación propios de las instituciones.

Ahora bien, no es suficiente el hecho de que la educación formal cuente con las herramientas necesarias para establecer un modelo que considere las dimensiones afectivas, cognitivas y disposicionales. Este tipo de educación debe además preocuparse por materializarlo de forma tal que los resultados se midan en acciones ambientales que trasciendan a la sociedad de manera responsable.

Con esto, el reto de la educación formal o no formal dista del tiempo o lugar en donde se imparte y alcanza un límite superior que exige que a corto, mediano o largo plazo el asunto de la educación ambiental sea inherente al deseo de formación del individuo, ( en tanto acto de responsabilidad social de la educación). Siendo así, es prioritario entender que en la educación ambiental debe primar la asociación, bien sea de la formación o la información, con una cadena de valores, actitudes y conceptos ligados a su vez a los intereses sociales.

Entendido esto, se debe rechazar aquella percepción común que asocia la información con lo informal y la formación con los procesos más estrictos. $E l$ Libro Blanco de la Educación Ambiental incluye a la información y la formación como instrumentos de la Educación ambiental; define que «a través de la información, se trata de dar a conocer hechos, situaciones o procesos, haciéndolos llegar al público de forma comprensible» (1999: ---) y argumenta que la formación se relaciona con un enfoque educativo integrador que concilia lo cognitivo y lo afectivo. Para los autores, una acción educativa exitosa requiere tener en cuenta las concepciones, conocimientos, valores y comportamientos previos de las personas. 


\section{Actividad y acción}

Alfie (2003) cita a Giordian para afirmar que «[n]o basta incorporar la naturaleza al conocimiento para transformar la naturaleza del conocimiento»(2003:88). Esta es una premisa fundamental que distancia el «activismo» de la acción, diferencia que radica en el hecho de que la acción se enmarca en un espacio de formación integral, es decir que abarca el ambiente desde su naturaleza, los conceptos y las consecuencias de la relación de los elementos del mismo. Por lo tanto no pretende a través de unos conceptos brindados en cátedras o «sensibilizaciones espejo» (catastrofización de las relaciones del ambiente) transformar la sincronía natural del conocimiento del ambiente. Partiendo de esto, el postulado que plantea este artículo establece que las acciones se derivan de la formación y la formación a su vez no ocurre solamente en espacios académicos sino que responde a un sinnúmero de situaciones y experiencias que se consolidan a lo largo de los años y que la educación está llamada a orientar en el sentido correcto.

Mientras, la actividad queda relegada a una expresión de un colectivo o individuo cuyo fin frente al ambiente es benéfico pero no suficiente para modificar las conductas; precisamente por el efecto desarticulado de su ejecución. La actividad ambiental, promovida generalmente desde colectivos reunidos por causas comunes con sentido altruista y responsable, genera movimientos que buscan transformaciones inmediatas con un afán implícito, pero no logran interacciones más allá de la convicción propia de quien dirige y consolida la actividad. Con esto no se pervierte el buen sentido de la actividad pero sí se descuida la perspectiva de formación. Bien lo define Sepúlveda al referirse a los procesos educativos ambientales explicando que estos «requieren el desarrollo de la capacidad de analizar críticamente y desde una perspectiva interdisciplinar los diferentes fenómenos y situaciones socio-culturalesambientales» (2009: ---). Entonces, orientar los contenidos ambientales desde el activismo no permite identificar los elementos propios de las situaciones ambiéntales porque no analiza desde su origen todos los factores inmersos, sino que limita a unos pocos su fundamento, copiando modelos de circunstancias ambientales similares pero de origen e interacción diferente.

En 2002 la Asociación Nacional de Universidades e Instituciones de Educación Superior de México concluyó en un estudio sobre la categorización de las acciones educativas relacionadas con el medio ambiente y el desarrollo sustentable en las IES en México, que las acciones se evidencian desde los roles administrativos, docentes, de investigación y proyección social. Esto señala de manera clara que las acciones en su sentido completo deben ser transversales y ligadas a lo misional de las instituciones, pero también deben ser planeadas por la dirección estratégica que define sus necesidades y proyectadas a través de los planes y proyectos incluidos (aquellos que tengan relación con la proyección social). Además, siendo consecuentes con lo formulado hasta 
ahora, deben acudir a los diagnósticos ambientales de los individuos y su comportamiento colectivo.

En este caso particular, las actividades derivaron de un proceso de acciones que a su vez se consolidaron desde la formación, completando así un ciclo ideal dentro de la educación ambiental; ciclo que la Política Nacional de Educación ambiental en Colombia plantea, pero que queda a veces inconcluso ante la autonomía universitaria y los intereses particulares de direccionamiento de las Instituciones, lo que desdibuja las intenciones de los PRAUS y otras alternativas de educación propuestas (Herrera et al., 2011).

La responsabilidad social de las Instituciones de Educación superior se refleja entre otras cosas en la actuación de sus egresados en el ámbito socialcultural-ambiental. En una revisión rápida de la incursión de la educación ambiental en el sector educativo superior se puede deducir que no es el eje trascendental de formación, o por lo menos no lo ha logrado ser a pesar de la intención de la política y la planeación. En su mayoría, la sensibilización se ha reducido al activismo ambiental. Aznar y Ull plantean que el profesional formado ambientalmente debe «vivenciar situaciones reales que propicien la reflexión sobre las dimensiones afectivas, estéticas y éticas de las relaciones interpersonales y con el medio natural y socio-cultural» (2009: ---). Estos autores, al igual que Sepúlveda (2009), rescatan la importancia de la formación ambiental y las acciones y actividades que de ella deriven.

De acuerdo con Castro y Balzaretti (2000), las actividades ambientales son óptimas en el desarrollo de la educación ambiental no formal porque no están ligadas necesariamente a la transformación de actitudes y significados de lo ambiental sino a la amplificación de un contenido ambiental que en el contexto puede ser tomado o desechado sin remordimiento por el beneficiado o participante de la actividad.

\section{El componente axiológico}

Las investigaciones en didáctica de las ciencias en las últimas décadas, han permitido establecer que los alumnos llegan a las aulas de clase con sus propias ideas acerca de cómo funciona el mundo. Esas representaciones no son aisladas sino que hacen parte de una compleja estructura conceptual, metodológica, actitudinal y axiológica. (Gallego y Pérez, 1994). En este mismo sentido, las experiencias de aprendizaje significan para el estudiante situaciones de cambio holístico que involucra el nivel conceptual, metodológico, actitudinal y axiológico.

En el ámbito de la educación ambiental (EA) en términos de formación, tal como se ha explicado anteriormente y atendiendo lo propuesto por Vega y Álvarez (2005), se ha promovido la gestión racional de los recursos y se ha 
consolidado, de alguna manera, la construcción tanto de conocimientos como de actitudes en beneficio de la naturaleza, asumiendo simultáneamente valores que deben propender por una sociedad ecológicamente equilibrada y sostenible.

Los autores antes mencionados esbozan de manera clara lo que ellos denominan el marco de referencia del modelo de EA que en alguna medida lograría contribuir con la denominada «alfabetización ambiental» o lo que en este escrito se entiende como formación ambiental. En el modelo en mención, la EA se conceptualiza desde dos tópicos diferente. El primero de ellos centrado en la base teórica en la que se abordan aspectos como la visión sistémica, la complejidad, la globalización, el desarrollo sostenible y la capacidad para pasar de la sensibilización a la acción. Y el otro centrado en los elementos didácticos como el aporte que debe hacer la EA para evidenciar la problemática ambiental y las causas que la originan, la capacitación para dar solución a los problemas ambientales existentes y futuros, la responsabilidad entendida como el sentir los problemas ambientales como propios con el fin de actuar en consecuencia y, por último, las metodologías y todo tipo de recursos acordes con estos planteamientos.

Atendiendo a lo propuesto por Campaner y De Longhi (2007), una visión holística de la formación incluye los aspectos antes mencionados y redimensiona la didáctica de la EA. La inclusión de conceptos, significados y problemáticas ambientales en los currículos no es ahora el eje de la problemática de la EA.

En este momento lo que se debe discutir es la didáctica de ese complejo concepto de formación ambiental, por lo que resulta prioritario encaminar las prácticas educativas para que se consoliden en directrices que refuercen el conocimiento sobre el desarrollo de actitudes y valores ambientales. Estos, unidos con significados claros y coherentes, permiten contar en un futuro con «ciudadanos» que sean profesionales respetuosos de las acciones y las decisiones que tomen a lo largo de sus vidas en cuanto a lo ambiental.

De igual manera, los autores antes mencionados hacen énfasis en la argumentación y en la rigurosidad del lenguaje científico, en el cual se encasilla el lenguaje ambiental. Igualmente enfatizan la necesidad de hacer uso de la estrategia didáctica argumentativa como recurso para la construcción de significados que les permitan a los estudiantes plantear sus propias posturas ante diferentes preguntas y situaciones de índole ambiental.

Redondeando, lo hasta aquí planteado respecto a la formación ambiental no puede limitarse exclusivamente al compendio conceptual sino que debe trascender a lo más íntimo de las personas, para transformar con intención el componente axiológico y afectivo y sin dejar de lado la rigurosidad de los conceptos naturales y científcos que le atañen a su propia naturaleza. 
El descuido de cualquiera de las partes (conceptual, actitudinal, afectiva ó axiológica) desencadena lo que hemos denominado activismo ambiental.

En cuanto a las implicaciones didácticas, las consideraciones anteriores conllevan a la renovación pedagógica y didáctica dentro del contexto de la libertad en la enseñanza propia de los maestros, pero sin desligarse de los tres campos ya mencionados y permitiendo el desencadenamiento de acciones ambientales que respondan a las necesidades y dinámicas de contexto social.

De esta manera, lo que se espera es propiciar el debate acerca de cómo fomentar una formación ambiental que posibilite la actitud creativa en los miembros de las comunidades educativas para así expresar y generar nuevos comportamientos ambientales favorables cuando se someten a condiciones fuera del contexto personal.

\section{Referencias bibliográficas}

Alfie, M, 2003. «Medio ambiente y universidad: retos y desafíos ambientales en la Universidad Autónoma Metropolitana-Azcapotzalco». El Cotidiano. 122 (19): 86-92.

Ángel, M. et al. 2009. «Valoración del cambio de actitudes hacia el medio ambiente producido por el programa didáctico "EICEA" en los alumnos de Educación Secundaria Obligatoria (14-16 años)». Revista Electrónica de Enseñanza de las Ciencias. 8 (3): 1019-1036.

Aznar, M. P. y M. A. Ull. 2009. «Education in basic competences for sustainable development. The role of University». Revista de Educación. Número extraordinario: 219-237.

Campaner, G. y A. L. De Longhi. 2007. «La argumentación en Educación Ambiental. Una estrategia didáctica para la escuela media». Revista Electrónica de Enseñanza de las Ciencias. 6 (2): 442-456.

Capdevila, I. 1999 «L'ambientalització de la universitat». Colección de Monografies d'Educació Ambiental. 6:---.

Castro, E. y K. Balzaretti. 2000. La educación ambiental no formal, posibilidades $y$ alcances [versión electrónica]. Tomado el --- de http://educar.jalisco.gob. $\mathrm{mx} / 13 / 13$ Auror.html consultado el 12072010.

Chawla, L. 1999. "Life paths into effective environmental action». The Journal of Environmental Education. 31 (1): 15-26. 


\section{Liliana Caycedo y Sonia Maria Rosas}

El valor de las acciones ambientales y su evidencia en el contexto formativo, artículo producto de la investigación

García, S. S. (Ed.-Coord.). 2005. Educación ecológica: reflexión y praxis en torno a la sequía en Chihuahua. México D.F.: Instituto de Ecología, A. C.

García Mira, R. y Real Deus, J. E. 2003. «People Enviroment interaction and Enviromental Action An introduction». Medio Ambiente y Comportamiento Humano. 4 (1): 1-3.

Gallego, R. y R. Pérez. 1994. Representaciones y conceptos científicos. Un programa de investigación. Bogotá: Departamento de Química UPN.

Gomera Martínez, A. 2008. La conciencia ambiental como herramienta para la educación ambiental: conclusiones y reflexiones de un estudio en el ámbito universitario. Tesis de doctorado en estudios avanzados en la línea de educación ambiental, Universidad de Córdoba.

Herrera, J. et al. 2011. «Políticas para la formación en lo ambiental, el caso de las universidades de Bogotá». Documento final convocatoria interuniversitaria Grupo INVESTIGARE. Publicado en Boletín INVESTIGARE 2011.

Ministerio de Ambiente de España. 1999. Libro Blanco de La Educación Ambiental En España [versión electrónica]. Tomado el 12 de julio de 2011 de http://www.oei.es/ decada/portadas/blanco.pdf.

Sepúlveda, L .E. 2009. «Una evaluación de los procesos educativo-ambientales de Manizales». Revista Luna Azul. 28: 46-56.

Vega, M. y P. Álvarez 2005. «Planteamiento de un marco teórico de la Educación Ambiental para un desarrollo sostenible». Revista Electrónica de Enseñanza de las Ciencias 4 (1): 1-16. 